\title{
The nebula around the post-AGB star 89 Herculis ${ }^{\star}$
}

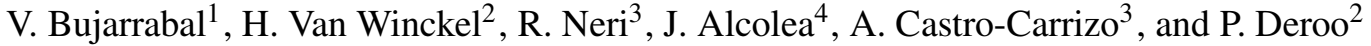 \\ 1 Observatorio Astronómico Nacional (OAN-IGN), Apartado 112, 28803 Alcalá de Henares, Spain \\ e-mail: v.bujarrabal@oan.es \\ 2 Instituut voor Sterrenkunde, KU Leuven, Celestijnenlaan 200B, 3001 Leuven, Belgium \\ e-mail: [Pieter.Deroo; Hans.VanWinckel]@ster.kuleuven.be \\ 3 Institut de Radio Astronomie Millimétrique (IRAM), 300 rue de la Piscine, 38406 St. Martin d'Hères, France \\ e-mail: [ccarrizo;neri]@iram.fr \\ 4 Observatorio Astronómico Nacional (OAN-IGN), C/ Alfonso XII 3, 28014 Madrid, Spain \\ e-mail: j . alcolea@oan.es
}

Received 19 December 2006 / Accepted 24 February 2007

\begin{abstract}
Aims. We aim to study the structure of the nebula around the post-AGB, binary star 89 Her. The presence of a rotating disk around this star had been proposed but not been yet confirmed by observations.

Methods. We present high-resolution PdBI maps of CO $J=2-1$ and 1-0. Properties of the nebula are directly derived from the data and model fitting. We also present $\mathrm{N}$-band interferometric data on the extent of the hot dust emission, obtained with the VLTI. Results. Two nebular components are found: (a) an extended hour-glass-like structure, with expansion velocities of $\sim 7 \mathrm{~km} \mathrm{~s}^{-1}$ and a total mass $\sim 3 \times 10^{-3} M_{\odot}$, and (b) an unresolved very compact component, smaller than $\sim 0{ }^{\prime} 4$ and with a low total velocity dispersion of $\sim 5 \mathrm{~km} \mathrm{~s}^{-1}$. We cannot determine the velocity field in the compact component, but we argue that it can hardly be in expansion, since this would require too recent and too sudden an ejection of mass. On the other hand, assuming that this component is a Keplerian disk, we derive disk properties that are compatible with expectations for such a structure; in particular, the size of the rotating gas disk should be very similar to the extent of the hot dust component from our VLTI data. Assuming that the equator of the extended nebula coincides with the binary orbital plane, we provide new results on the companion star mass and orbit.
\end{abstract}

Key words. stars: AGB and post-AGB - stars: winds, outflows - radio lines: stars - stars: individual: 89 Her

\section{Introduction}

Planetary and protoplanetary nebulae (PNe, PPNe) very often show axisymmetric shapes and fast axial expansion, which are thought to be due to shock interaction between the very collimated post-AGB jets and the slow and isotropic AGB wind. Theoretical calculations show that accretion from rotating disks onto the post-AGB star or a companion can explain the axial jets (see Soker 2002; Frank \& Blackman 2004, etc.) and provide the high energy and momentum required to explain the PPN dynamics (Bujarrabal et al. 2001). On the other hand, the presence of a large amount of orbiting material has been invoked to explain some remarkable observational properties: 1) the peculiar abundances sometimes found in the atmospheres of post-AGB stars, where refractory elements are deficient, presumably because of reaccretion of material in which grains have been efficiently formed (removing such elements from the gas) and expelled (Van Winckel 2003); and 2) the detection of a NIR excess due to dust at temperatures close to that of sublimation, which should correspond to stable structures (i.e. in Keplerian rotation) close to the star. Such SEDs are rather common among post-AGB stars

* Based on observations carried out with the IRAM Plateau de Bure Interferometer, as well as on observations of the Belgian Guaranteed time on VISA (ESO). IRAM is supported by INSU/CNRS (France), MPG (Germany) and IGN (Spain). and, in a review paper of de Ruyter et al. (2006), fifty one objects are listed. These features are systematically found to be associated to binarity, which would explain the excess of angular momentum needed to form Keplerian disks from previously ejected gas.

The existence of rotating disks around post-AGB stars is therefore a basic question to understand the post-AGB evolution, in particular, the shape and dynamics of PPNe.

Disks or tori of molecular gas around post-AGB stars are commonly detected as the central part of PPNe, although in general they are not observed to rotate, but to be in expansion, like the rest of the nebula. It has been suggested (e.g. Fong et al. 2006) that wide oblate structures found around a few AGB stars could be in rotation, but the evidence is very controversial (Josselin et al. 2000; Hirano et al. 2004, etc). Direct detection of a Keplerian velocity field has been obtained for only one PPN till now: the Red Rectangle (Bujarrabal et al. 2005), a well-known PPN surrounding a binary star.

89 Her (HR 6685) was first studied by Bidelman (1951), who noted the presence of a presumably massive supergiant at high Galactic latitude. There is now, however, general consensus that $89 \mathrm{Her}$ is a low-mass object in its post-AGB phase of evolution. It is one of the few post-AGB stars with a significant Hipparcos parallax, placing it at $1_{0.6}^{2.3} \mathrm{kpc}$ from the Sun. Interestingly, $89 \mathrm{Her}$ is a binary system that shows both a conspicuous NIR excess, 

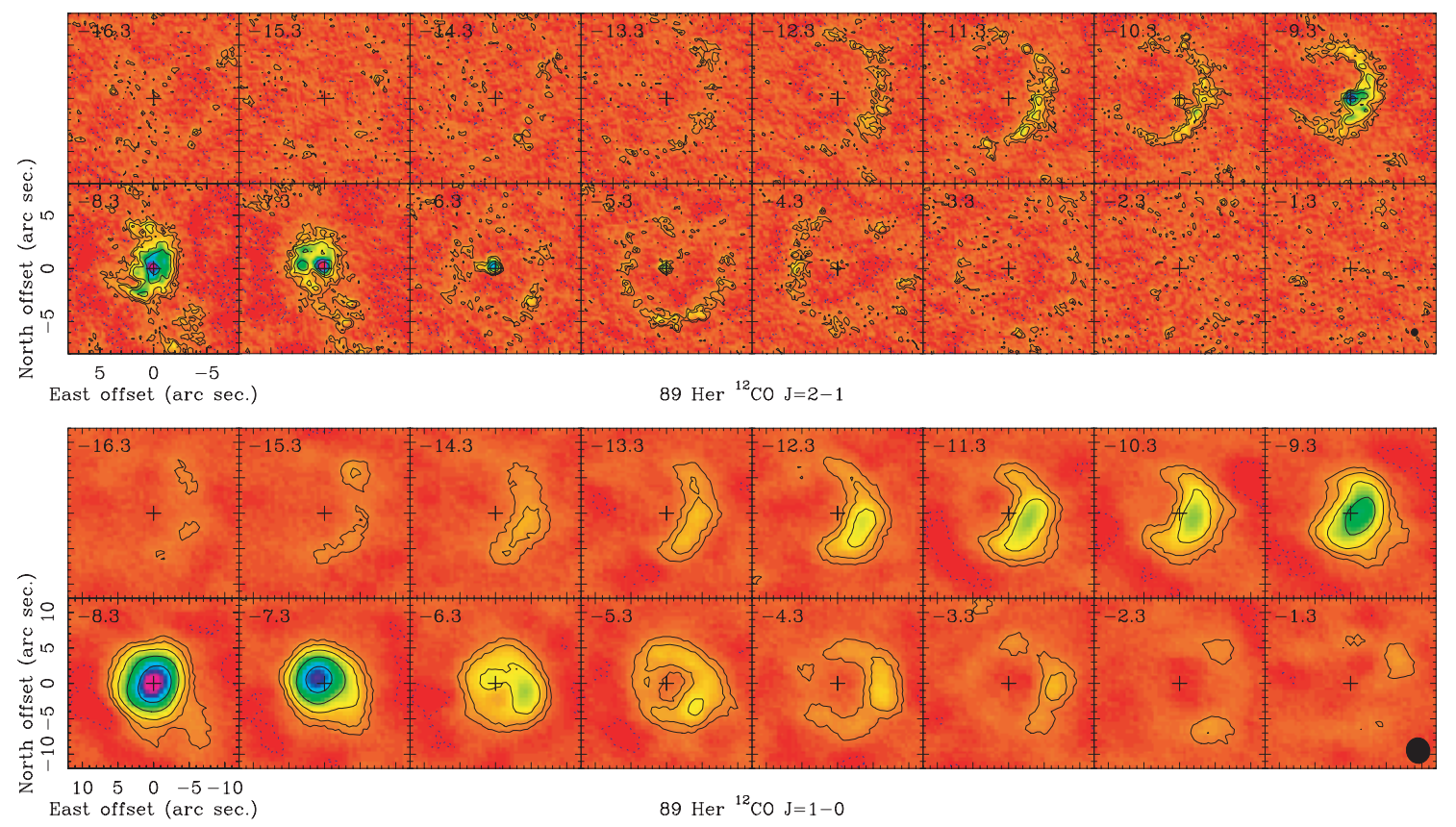

Fig. 1. Channel maps of the ${ }^{12} \mathrm{CO} J=2-1$ (upper panels) and $J=1-0$ lines (lower panels) from 89 Her. The first contours are, respectively, 15 and $30 \mathrm{mJy}$; the contours are separated by a factor 2 and the negative contours (at -15 and $-30 \mathrm{mJy}$, respectively) are indicated by dashed lines. The LSR velocity in $\mathrm{km} \mathrm{s}^{-1}$ for the center of each channel is indicated in the upper left corner. The J2000 coordinates for the reference position, the cross in the maps, are RA 15:55:25.19 and Dec +26:03:00.0. The black ellipses in the last panels indicate the beam half-intensity sizes.

due to hot dust (Waters et al. 1993; de Ruyter et al. 2006), and a far-IR flux indicating the presence of large grains (Shenton et al. 1995). Its CO lines show a peculiarly sharp profile very similar to the one detected in the Red Rectangle (Bujarrabal et al. 2001). Previous interferometric maps of the CO emission from 89 Her (Alcolea \& Bujarrabal 1995; Fong et al. 2006) have not yielded conclusive results, due to the small size and low intensity of the source. A roughly rounded structure was found, showing a central core plus an extended clumpy halo, with no clear velocity pattern.

\section{Observations}

We report on high-resolution interferometry with the IRAM Plateau de Bure Interferometer of the ${ }^{12} \mathrm{CO}(1-0)$ and ${ }^{12} \mathrm{CO}(2-1)$ lines and continuum emission from 89 Her. Observations were carried out under excellent atmospheric conditions (pwv = $0.5-2 \mathrm{~mm}$, seeing $\simeq 0{ }^{\prime} 1-0$. $^{\prime} 4$ ) with the six-element array in the D configuration (November 11, 2005) and in the newly extended A configuration (January 19 and 25, 2006). The cross-correlator was set up to cover the lines with 20 and $40 \mathrm{MHz}$ bands each and the continuum emission with two $320 \mathrm{MHz}$ bands. The flux calibration was referred to MWC349 and 3C273, while phase and amplitude calibrations were done using $1751+288$.

Data were calibrated in the antenna-based manner using the GILDAS ${ }^{1}$ software package. Velocity maps were produced with natural weights at $2.6 \mathrm{~mm}$ in order to analyze the extended molecular distribution and by applying an inverse Gaussian taper at $1.3 \mathrm{~mm}$ to analyze in detail the compact emission in the immediate surroundings of $89 \mathrm{Her}$. Cleaned and primary beam-corrected maps were obtained with synthesized beams of $3^{\prime \prime} .60 \times 33^{\prime \prime} .32$ at position angle $-97^{\circ}$ and $0 .^{\prime} 68 \times 0 .{ }^{\prime} 60$ at $-97^{\circ}$, respectively at $2.6 \mathrm{~mm}$ and $1.3 \mathrm{~mm}$.

1 The GILDAS software package is available for download on the IRAM web pages.
The line emission was resampled to an effective velocity resolution of $1 \mathrm{~km} \mathrm{~s}^{-1}$ yielding a one-sigma sensitivity level of $\sim 9.3 \mathrm{mJy} /$ beam for the ${ }^{12} \mathrm{CO}(1-0)$ and of $\sim 15.3 \mathrm{mJy} / \mathrm{beam}$ for the ${ }^{12} \mathrm{CO}(2-1)$ line. The continuum sensitivities were estimated from the line-free emission regions of the bands to be $\sim 0.3 \mathrm{mJy} /$ beam at $2.6 \mathrm{~mm}$ and $\sim 0.5 \mathrm{mJy} /$ beam at $1.3 \mathrm{~mm}$. Continuum emission was well-detected at $2.6 \mathrm{~mm}$ and $1.3 \mathrm{~mm}$ but remained unresolved, yielding flux densities of $2.7 \mathrm{mJy}$ and $9.2 \mathrm{mJy}$, respectively, compatible with thermal dust emission. The size of the continuum source is smaller than 0.3 .

\subsection{VLTI data}

We also performed N-band interferometric observations using the two-beam VLTI/MIDI instrument. Three set-ups were used in April 2006 during Belgian guaranteed time on the auxiliary telescopes (VISA). For one of the observations, the baseline of $16 \mathrm{~m}$ (projected angle $=78$ degrees) was used, while the other two were conducted on a larger baseline of $32 \mathrm{~m}$ with similar projected angles (80, 77 degrees). The visibilities were calibrated using observations of unresolved calibration stars (HD 168454 and HD 123139), which were observed in strict concatenation with the science target. These observations were performed in the context of a large program to systematically study the circumstellar discs around binary post-AGB stars. With the $1.8 \mathrm{~m}$ auxiliary telescopes, the sensitivity of MIDI allowed fringe detection on a correlated flux larger than $20 \mathrm{Jy}$ at $12 \mu \mathrm{m}$. For the details on the data reduction, we refer the reader to Deroo et al. (2006).

\section{Nebula structure: a rotating disk around 89 Her?}

As seen in our maps (Fig. 1), the nebula around 89 Her consists of two very different features, a quite extended one and a very compact central clump. The shape of the extended component strongly suggests an expanding hour-glass-like structure, 
whose axis is slightly inclined with respect to the line of sight. The extended component must have a maximum expansion velocity $\sim 6-7 \mathrm{~km} \mathrm{~s}^{-1}$ (the velocity probably increases with the distance to the star, in view of the variations in the image size with the velocity and as is confirmed by the model calculations explained below). For a distance of $1 \mathrm{kpc}$ (Sect. 1), the typical total size of this extended component is estimated to be $\sim 1.5 \times 10^{17} \mathrm{~cm}$.

We do not detect the extent of the compact component, which must be smaller than $\sim 0{ }^{\prime} 4$, equivalent to a diameter smaller than $\sim 5 \times 10^{15} \mathrm{~cm}$. This size limit is comparable to that of the dust emitting at $1 \mathrm{~mm}$ wavelength, and probably both emissions come from the same component. Note that interferometric maps of the optically-thin dust emission at this wavelength strongly select the massive compact components. The total velocity dispersion is $\sim 5 \mathrm{~km} \mathrm{~s}^{-1}$, with no significant velocity pattern. A slight velocity gradient in the north-east south-west direction could be seen in our $J=2-1$ maps of the central clump, which could be consistent with the trend found for the extended component. But note that contamination by the emission of the wide component may be relevant in this respect, as it happens in the $J=1-0$ maps, where the extended component emission always dominates the brightness distribution due to the relatively wide beam. An inner E-W elongation also appears in the lowresolution maps by Fong et al. (2006), but our data clearly show that it is due to emission from the hour-glass-like structure.

We have tried to reproduce our data by synthetic maps from an axially symmetric model nebula. The model description and assumptions can be found elsewhere (e.g. Bujarrabal et al. 1998; Castro-Carrizo et al. 2002). We considered an hour-glass-like extended structure, with very thin walls, plus an equatorial compact disk.

In Fig. 2 we represent the model nebula shape that reproduces our data, as well as the density and velocity distributions in the extended component. The symmetry axis of this component forms an angle of $\sim 15^{\circ}$ with the line of sight, i.e. 75 degrees with respect to the plane of the sky. In the extended component, the total density is assumed to be inversely proportional to the distance to the star, $r$, with $n\left(10^{16} \mathrm{~cm}\right)=3 \times 10^{3} \mathrm{~cm}^{-3}$. The rotational temperature of the extended nebula decreases linearly with $r$, between $11 \mathrm{~K}$ and $5 \mathrm{~K}$. The velocity in this component is radial and its modulus increases linearly with $r$, between $2.5 \mathrm{~km} \mathrm{~s}^{-1}$ in the center up to $8 \mathrm{~km} \mathrm{~s}^{-1}$. The $\mathrm{CO}$ relative abundance is assumed to be constant and equal to $3 \times 10^{-4}$ in all the nebula. Note that we have tried to keep the model very simple, in view of the lack of observational information.

The values of most parameters of the central unresolved component are uncertain. The velocity field of the compact disk cannot be reliably deduced from the data, as mentioned above, so only a velocity dispersion can be estimated for this component. We have taken conditions for which the ${ }^{12} \mathrm{CO}$ lines are optically thick, in view of the low contrast shown in the line core for ${ }^{12} \mathrm{CO}$ and ${ }^{13} \mathrm{CO}$ transitions (Bujarrabal et al. 2001). Therefore, ${ }^{12} \mathrm{CO}$ (and even ${ }^{13} \mathrm{CO}$ ) lines could be opaque in it, and the derived density and mass in the central clump are only lower limits. Assuming high optical depth and the size limit given above, we can derive a kinetic temperature limit from the measured brightness, $T_{k}>60 \mathrm{~K}$.

The total masses of both components are compatible with those derived in Bujarrabal et al. (2001) from ${ }^{13} \mathrm{CO}$ lines $\left(\sim 10^{-2} M_{\odot}\right.$ for the central component and $\sim 3 \times 10^{-3} M_{\odot}$ for the extended one; note that we must correct the mass values in that paper for the different assumed distance).

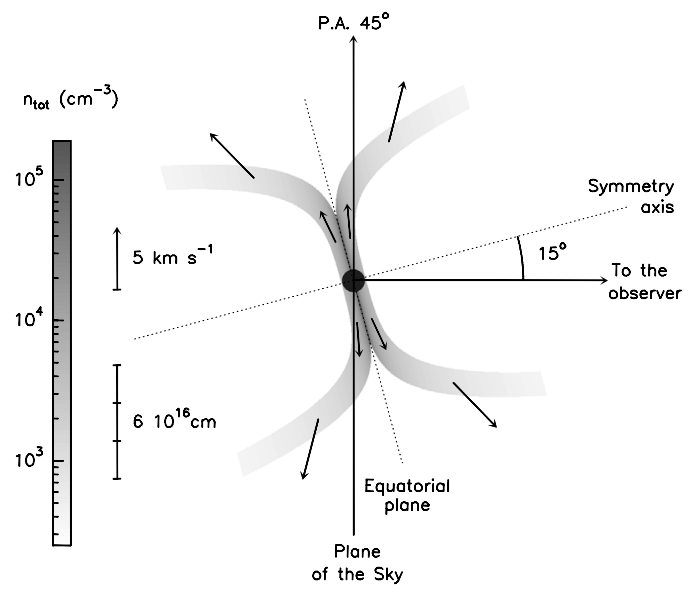

Fig. 2. Geometry and distribution of density and velocity in the model nebula. Note that we only obtained limits for the size and physical conditions of the central, compact component, represented by the black circle.

The best fitting of the $J=2-1$ maps from our model is found in Fig. 3. Note that the model necessarily predicts quite symmetric results for relatively positive and negative velocities, with respect to the equator, which is not exactly the case in the observations. Therefore, our fitting can only represent a kind of average of the maps around the central velocities. The observed asymmetry could correspond to a small deviation of the symmetry axis for both cups with respect to the line of sight, by about $10 \mathrm{de}-$ grees; a similar deviation for the projection of the axis on the plane of the sky is also directly suggested by our maps.

From the size and velocity of the extended component given above, we find a typical lifetime $\sim 3500 \mathrm{yr}$. This value is high compared to the typical ages of other PPNe, often around $1000 \mathrm{yr}$, but compatible with expectations for post-AGB stars with low initial mass (e.g. Vassiliadis \& Wood 1994; Blöcker 1995).

If we also assume that the compact component is in expansion, we can calculate a typical lifetime. If, as often happens in bipolar nebulae, it is a disk perpendicular to the symmetry axis of the extended hour-glass structure (then forming an angle of about $15^{\circ}$ with respect to the plane of the sky), the lifetime must be shorter than $\sim 100 \mathrm{yr}$. A less strong upper limit is obtained if we assume that the central structure is spherical and in isotropic expansion, in this case the lifetime must be shorter than $300 \mathrm{yr}$.

Note the significant difference between the lifetimes of both extended and compact components. The very short lifetime derived for the compact core is surprising. We expect copious mass losses in AGB stars, but not that an F2-type star ejects about $10^{-2} M_{\odot}$ in such a sudden event, completely independent of the ejection of the extended component. Moreover, we note that the star is slowly evolving now. It is known to have its present efficient temperature since at least 1950 (see Waters et al. 1993; Bidelman 1951) up to 2005 (unpublished data), so we do not expect that it was in the AGB only $\sim 100 \mathrm{yr}$ ago.

The very inner nebular structure was also probed by our VLTI observations. The total N-band flux is completely dominated by the circumstellar hot dust component. Even on our longest projected baseline $(31.1 \mathrm{~m})$, this $\mathrm{N}$-band emission is barely resolved, with normalized visibility amplitudes $>0.7$ in the whole spectral band. The hot dust therefore must come from an angular size smaller than $\sim 31$ mas, i.e. a diameter $\lesssim 31 \mathrm{AU}$ at 


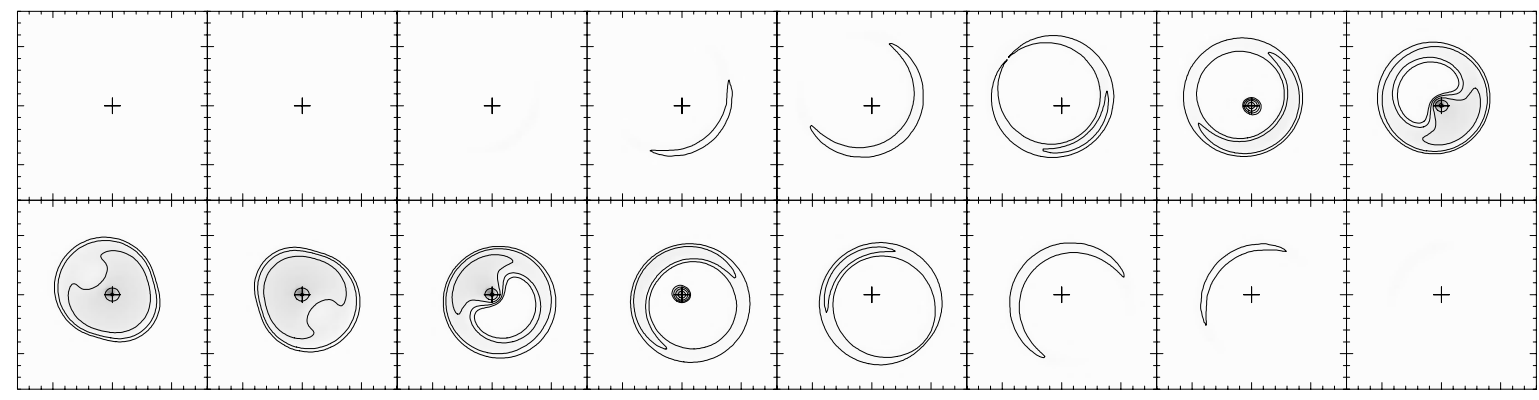

89 Her ${ }^{12} \mathrm{CO} \mathrm{J}=2-1$, model predictions

Fig. 3. Synthetic maps predicted by our model of the ${ }^{12} \mathrm{CO} J=2-1$ line emission from the nebula around 89 Her. Contours and units are the same as for Fig. 1.

the distance of 89 Her. This result is compatible with the unresolved N-band direct image by Meixner et al. (1999).

If we assume that the central component is a rotating disk, we cannot derive lifetimes, but we can compare the velocities with the Keplerian ones. Taking a typical projected velocity of $\sim 2 \mathrm{~km} \mathrm{~s}^{-1}$, the characteristic rotation velocity would be $\sim 8 \mathrm{~km} \mathrm{~s}^{-1}$ for the deduced axis inclination. For a central total mass of $1 M_{\odot}$ (see mass estimate in Sect. 4), the rotating gas must be at a typical distance of about $15 \mathrm{AU}$, yielding a typical disk size $\sim 4 \times 10^{14} \mathrm{~cm}$ or 30 milli-arcseconds. We note that the outer diameter of the rotating disk could be larger than these values, corresponding to lower rotation velocities but included within the observed velocity dispersion. This very compact size is strikingly compatible with the size measured from our VLT interferometry at $10 \mu \mathrm{m}$. We finally note that a typical temperature for the central disk $T_{k} \sim 600 \mathrm{~K}$ is derived from the measured brightness temperature (at relatively high velocities but within the central component) and a size $\sim 0$ ' $^{\prime} 05$. This value is comparable to the temperature of the warm dust derived from analysis of the SED in 89 Her and to that of the Keplerian disk in the Red Rectangle derived from CO emission modelling, $\sim 500 \mathrm{~K}$ at $4 \times 10^{14} \mathrm{~cm}$ (Bujarrabal et al. 2005).

\section{Discussion and conclusions}

Our CO data of 89 Her show the presence of a double structure, with an unresolved compact circumstellar component and an hour-glass-like extended outflow. Although the velocity field in the inner region is not well-measured, we conclude that it is probably a Keplerian disk with a very small extent (diameter $\left.\$ 10^{15} \mathrm{~cm}, \lessgtr 0 !^{\prime} 1\right)$. This is supported by our N-band interferometric data that shows that, in the N-band, the extent of the dust emission is barely resolved at a $31.1 \mathrm{~m}$ baseline. The dust emitting in the $1 \mathrm{~mm}$ continuum in $89 \mathrm{Her}$ is also confined to a small central region (diameter $<00^{\prime} 3$ ), probably the same compact component.

The existence of rotating inner disks has been proposed to explain some properties of hot dust emission from other binary post-AGB stars (Sect. 1), properties that are in fact shared by the Red Rectangle and 89 Her. Our results support the identification of inner stable reservoirs of material in Keplerian rotation in these objects. The small size of the Keplerian disk in 89 Her can be a common property of such candidate disks, in view of the high dust temperature measured in them. 89 Her and the Red Rectangle are likely the nearest of these objects (de Ruyter et al. 2006), so that these small regions are not expected to be resolved by present day radio interferometers, and a direct confirmation of the Keplerian dynamics in the other objects will require the use of future instruments. Our N-band interferometric experiments do confirm the very compact nature of the warm dust emission in similar objects like HD 52981 and SX Cen (Deroo et al. 2006).

Assuming that the symmetry axis of the resolved hour-glass outflow is perpendicular to the inner orbit of the binary, the mass function of $0.000838 M_{\odot}$ (Waters et al. 1993) converts to a mass of the companion of only $0.35 M_{\odot}\left(i=10^{\circ}\right.$ gives $0.62 M_{\odot}$, $i=20^{\circ}$ yields $0.25 M_{\odot}$ ) assuming a primary of $\sim 0.6 M_{\odot}$. Since there is no evidence of a hot, compact degenerate component in the system, the companion is likely to be a very low-mass main sequence dwarf.

With this inclination the orbit has a semi-major axis of $0.31 \mathrm{AU}(a \sin i=0.08 \mathrm{AU})$. This orbit is well within the sublimation radius of the primary, so the circumstellar dusty disk must be circumbinary. The primary does not fill its Roche-lobe now; but assuming the star had a similar luminosity when on the AGB, it must have had a phase of very strong binary interaction.

Acknowledgements. J.A. and V.B. acknowledge partial support from the Spanish Ministry of Education $\mathcal{E}$ Science project numbers AYA2003-7584 and ESP2003-04957

\section{References}

Alcolea, J., \& Bujarrabal, V. 1995, A\&A, 303, L21

Bidelman, W. P. 1951, ApJ, 113, 304

Blöcker, T. 1995, A\&A, 299, 755

Bujarrabal, V., Alcolea, J., \& Neri, R. 1998, ApJ, 504, 915

Bujarrabal, V., Castro-Carrizo, A., Alcolea, J., \& Sánchez Contreras, C. 2001, A\&A, 377, 868

Bujarrabal, V., Castro-Carrizo, A., Alcolea, J., \& Neri, R. 2005, A\&A, 441, 1031 Castro-Carrizo, A., Bujarrabal, V., Sánchez Contreras, C., Alcolea, J., \& Neri, R. 2002, A\&A, 386, 633

de Ruyter, S., Van Winckel, H., Maas, T., et al. 2006, A\&A, 448, 641

Deroo, P., Van Winckel, H., Min, M., et al. 2006, A\&A, 450, 181

Fong, D., Meixner, M., Sutton, E. C., Zalucha, A., \& Welch, W. J. 2006, ApJ, 652,1626

Frank, A., \& Blackman, E. G. 2004, ApJ, 614, 737

Hirano, N., Shinnaga, H., Dinh-V-Trung, et al. 2004, ApJ, 616, L43

Josselin, E., Mauron, N., Planesas, P., \& Bachiller, R. 2000, A\&A, 362, 255

Meixner, M., Ueta, T., Dayal, A., et al. 1999, ApJS, 122, 221

Shenton, M., Evans, A., \& Williams, P. M. 1995, MNRAS, 273, 906

Soker, N. 2002, ApJ, 568, 726

Van Winckel, H. 2003, ARA\&A, 41, 391

Vassiliadis, E., \& Wood, P. R. 1994, ApJS, 92, 125

Waters, L. B. F. M., Waelkens, C., Mayor, M., \& Trams, N. R. 1993, A\&A, 269, 242 